\title{
Current research for a vaccine against Lassa hemorrhagic fever virus
}

This article was published in the following Dove Press journal:

Drug Design, Development and Therapy

\author{
Bryce M Warner' \\ David Safronetz ${ }^{2}$ \\ Derek R Stein ${ }^{2}$ \\ 'Department of Medical Microbiology, \\ University of Manitoba, Winnipeg, \\ MB, Canada; ${ }^{2}$ Zoonotic Diseases and \\ Special Pathogens Program, National \\ Microbiology Laboratory, Public \\ Health Agency of Canada, Winnipeg, \\ MB, Canada
}

\begin{abstract}
Lassa virus (LASV) is a rodent-borne arenavirus endemic to several West African countries that causes Lassa fever (LF). LF is typically mild but it can cause severe disease characterized by hemorrhagic fever and multi-organ failure. A current outbreak of LASV in Nigeria has seen greater than 300 cases with a case fatality rate of $22 \%$. Currently, there are limited treatment options and no vaccine candidates are approved to prevent LASV infection. The Coalition for Epidemic Preparedness Innovations has identified LASV as an emerging pathogen of high consequence and this has resulted in a push for several preclinical vaccine candidates to be advanced toward clinical trials. Here, we discuss several important aspects of LASV infection including immunobiology, immune evasion, and correlates of protection against LF, which have been identified through animal models and human infections. In addition, we discuss several vaccine candidates that have shown efficacy in animal models that could be advanced toward clinical trials. The increased fatality rate seen in the recent LASV outbreak in Nigeria highlights the importance of developing effective treatment and prevention strategies against LF. The spike in LASV cases seen in West Africa has the potential for increased mortality and human-to-human transmission, making the development and testing of effective vaccines for LASV critical.
\end{abstract}

Keywords: Lassa virus, Lassa fever, vaccine, therapeutics, prevention, pathogenesis

\section{Introduction}

Nigeria is currently struggling with a severe reoccurrence of Lassa fever (LF), with confirmed cases in over half of the country (18 States). ${ }^{1}$ The World Health Organization (WHO) is currently reporting thousands of suspected cases with 317 confirmed cases, over 72 deaths, and a probable case fatality rate of $22 \%$. In addition, 14 health care workers have been infected with a case fatality rate of $30 \%$. LASV was first discovered in 1969 when two missionary nurses died in Nigeria. ${ }^{2}$ Lassa virus (LASV), the causative agent of LF, belongs to the family Arenaviridae, and is endemic over most of West Africa (Figure 1). It is transmitted by the multimammate rat, Mastomys natalensis, ${ }^{3}$ and has an enveloped, negative-sense, RNA genome. The genome is ambisense and consists of two segments, a small (S) segment and a large (L) segment. The S segment encodes the glycoprotein precursor (GPC), which is expressed on the envelope of the virus in a trimeric state. ${ }^{4}$ The $\mathrm{S}$ segment also encodes the nucleoprotein (NP) in the opposite direction, which encapsulates the viral genome. The L segment encodes the viral matrix protein (Z) and the viral RNA-dependent RNA polymerase (L). When transmitted to humans, LASV presents in most cases (80\%) as a mild non-descript disease; however, $20 \%$ of infections result in severe hemorrhagic fever with multi-organ failure. ${ }^{5}$ The incubation period of LF is typically 1-3 weeks with accompanying headache, fever, muscle/joint pain, diarrhea, vomiting, elevated liver enzymes (AST and ALT), and
Correspondence: Derek R Stein

Zoonotic Diseases and Special Pathogens

Program, National Microbiology

Laboratory, Public Health Agency

of Canada, I0I5 Arlington Street,

Winnipeg MB R3E 3R2, Canada

$\mathrm{Tel}+$ | 2044701910

Email derek.stein@canada.ca
Drug Design, Development and Therapy 2018:12 25/9-2527

2519

Dovepress f $y$ in 0

http://dx.doi.org/10.21471DDDTS147276

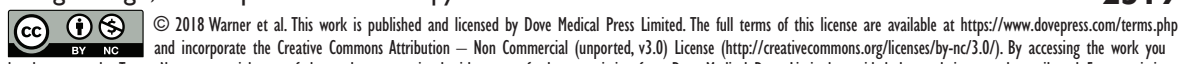
hereby accept the Terms. Non-commercial uses of the work are permitted without any further permission from Dove Medical Press Limited, provided the work is properly attributed. For permission for commercial use of this work, please see paragraphs 4.2 and 5 of our Terms (https://www.dovepress.com/terms.php). 


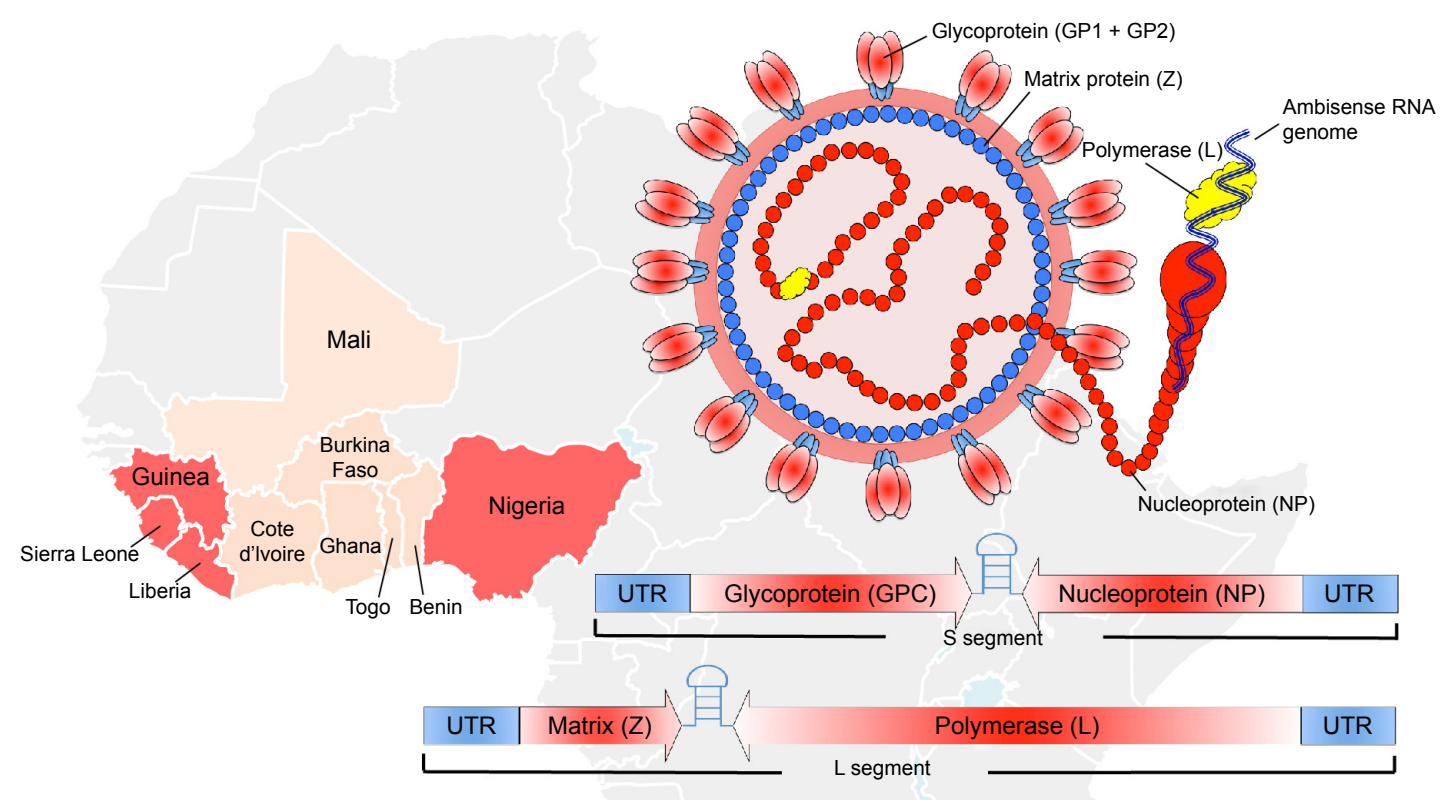

Figure I Lassa virus geographical distribution and virus structure.

Notes: Lassa virus has caused numerous outbreaks and is endemic to Nigeria, Guinea, Sierra Leone, and Liberia (red). There is also strong evidence of Lassa virus infections (beige) throughout much of West Africa (WHO Lassa Report, 201866). The Lassa virus structure and genome are also depicted.

Abbreviations: RNA, ribonucleic acid; UTR, Untranslated Region; WHO, World Health Organization.

hematocrit. The signs of pathogenesis can vary depending on the animal model used in various vaccine studies; however, the gold standard that best recapitulates the human condition are non-human primates (NHPs) (Figure 2A). A poor prognosis is also indicated with accompanying edema of the face and neck, abdominal and retrosternal pain, enlarged lymph nodes, and/or hemorrhage in the conjunctiva or mucosal surfaces. It is estimated that there are over 300,000 infections on an annual basis in West Africa, making LASV a primary cause of hemorrhagic fever worldwide. Currently, there are no approved vaccines or therapeutics for the treatment or prevention of LASV with the questionable exception of the off-label use of ribavirin. ${ }^{6}$ Additionally, historical imported cases of LASV infection and human-to-human transmission warrant strong development of vaccine and treatment modalities. ${ }^{5,7,8}$ Recently, the Bill and Melinda Gates Foundation along with its collaborating partners founded the Coalition for Epidemic Preparedness Innovations (CEPI) with the sole purpose of advancing three priority pathogens with promising preclinical candidates to Phase III clinical trials in the event of future outbreaks. CEPI, along with the WHO, has initially named LASV, Middle East Respiratory Syndrome Coronavirus, and Nipah virus as the top pathogens of priority for their first round of applications. Despite the high disease burden and lack of preventative measures for LASV, significant progress has been made in the identification of promising preclinical candidates. In this review, we discuss the current understanding of LASV immunobiology, the correlates of protection, and the most promising vaccine candidates for the prevention of LF.

\section{Immunology of Lassa virus infections Innate immune system}

Professional antigen-presenting cells (APC), such as macrophages and dendritic cells (DCs), are the primary target cells for LASV infection, and initial infection typically involves myeloid lineage cells and APC. ${ }^{9,10}$ An early determinant of disease progression in arenavirus infection is the activation status of virally infected APC. During LASV infection, APC fail to undergo activation or maturation, leading to ineffective antigen processing and presentation despite LASV being able to replicate at high levels within these cells. ${ }^{9}$ This is in contrast to Mopeia virus (MOPV), a non-pathogenic Mammarenavirus very similar to LASV, but one that induces APC activation leading to effective adaptive immunity while also replicating at high levels within APC. ${ }^{3}$ Infection with MOPV leads to classically activated APC with upregulation of CD80, CD86, and CD40, along with secretion of innate cytokines such as type I interferons (IFN) and interleukin- $6 .{ }^{11}$ The immature state of infected APC persists during LASV infection even though cells migrate to the draining lymph nodes of infected individuals. ${ }^{12}$

During early LASV infection, the type I IFN response has been shown to be an important mediator of protection 


\section{A Disease course:}

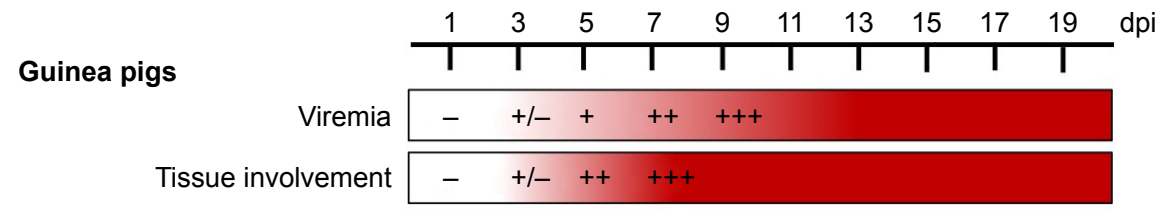

\section{Non-human primates}

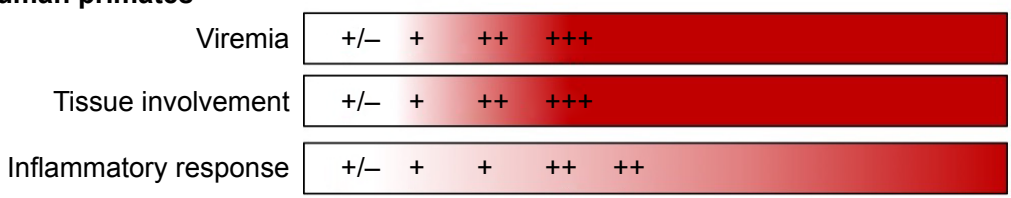

Humans*

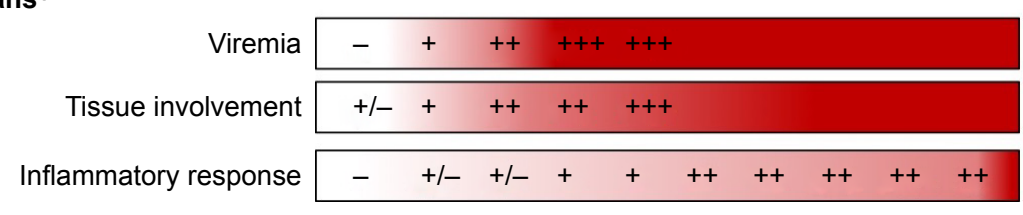

B Efficacy of vaccines and therapeutic interventions:

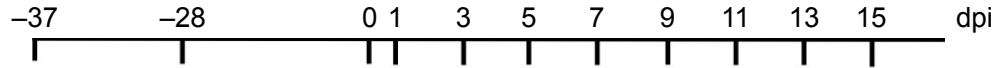

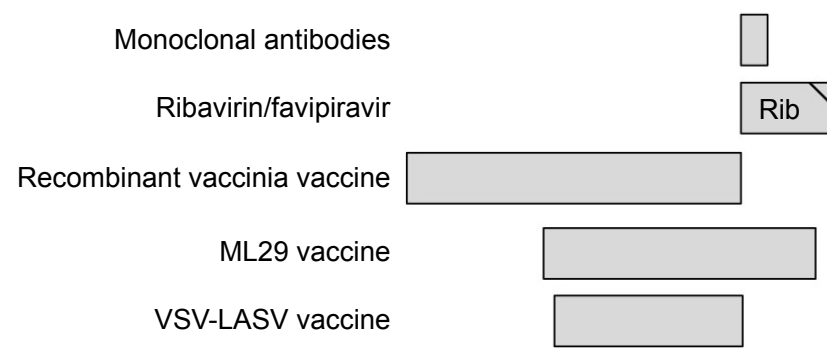

Figure 2 Disease course of Lassa virus infection and efficacy of various preventative strategies.

Notes: The timeline of various aspects of disease course during Lassa virus infection in the most commonly used animal models and in humans (A), and the timeline of administration of various preventative strategies against Lassa virus infection (B). Each of the vaccine strategies listed has shown protective efficacy in guinea pigs and/or NHPs when administered at the day listed relative to infection. *Following symptom onset.

Abbreviations: NHPs, non-human primates; Rib, ribavirin; Fav, favipiravir; VSV-LASV, vesicular stomatitis virus-Lassa virus.

against the virus. ${ }^{13}$ NHPs infected with LASV that have a strong IFN $\alpha$ response within the first few days of infection fare better than animals with minimal IFN $\alpha$ early on during infection. Additionally, pathogenic and lethal LF models have been characterized using mice lacking the IFN $\alpha / \beta$ receptor and thus unable to respond to IFN $\alpha$ or IFN $\beta$. This is in contrast to wild-type mice, which are resistant to LASV infection. ${ }^{14-16}$ Type I IFN signaling provides an important barrier to virus replication as well as a bridge to the virus-specific adaptive immune response. Therefore, the ability of LASV to alter the type I IFN response may allow for unchecked viral replication and an escape of protective adaptive immune responses. There is evidence that LASV can antagonize the innate IFN response and other cellular mechanisms that lead to both innate and adaptive immune responses, which may be critical for viral clearance and reduced pathogenesis. As already mentioned, LASV prevents APC activation and maturation, which is achieved through reduced RNA sensing in infected DCs. ${ }^{9}$ The $\mathrm{Z}$ protein of LASV also binds to the caspase recruitment domain of RIG-I and MDA5, preventing signaling by both proteins. ${ }^{17}$ The nucleocapsid protein of LASV can also inhibit type I IFN signaling by degrading dsRNA and by binding to I $\kappa$ B kinase- $\varepsilon$, preventing activation of IRF3 and NF- $\kappa B .{ }^{18,19}$ There is also evidence that LASV can use TAM receptors, AXL and TYRO3, for cell entry. ${ }^{20,21}$ The activation of these receptors leads to upregulation of suppressor cytokine signaling proteins and inhibition of type I IFN signaling pathways. LASV can use the abovementioned strategies to prevent RIG-I signaling, leading to type I IFN responses in infected cells. These strategies play 
an important role in preventing APC activation, leading to adaptive immune responses and viral clearance. The ability to alter the function of APC is likely a critical determinant of pathogenesis during LASV infection, as an absent adaptive immune response is typically seen in fatal LF cases. ${ }^{22,23}$ Overall, there is strong evidence that altered APC function during LASV infection correlates with a severe disease outcome. Knowledge of how LASV modulates the innate immune system can have important implications for the development of vaccines and therapeutics. While most vaccine candidates target the glycoprotein of LASV and thus only express this protein and not those known to modulate innate immunity, candidates such as the ML29 reassortant vaccine express LASV nucleocapsid protein. This may have important implications for the immunogenicity of this vaccine and other candidates that may include proteins from LASV with immunomodulatory properties.

\section{Adaptive immune system and correlates of protection}

The activation of LASV-specific T cells is thought to play an important role during infection as LF development results in the depletion of T cells in NHP models. ${ }^{13,24}$ Both CD4 and CD8 T cells can be activated early following LASV infection, and LASV-NP-specific memory CD4 T cells have been detected in patients up to 6 years following infection..$^{25,26}$ The activation of $\mathrm{T}$ cells during LASV infection requires APC activation and maturation for efficient antigen processing and presentation. As with infection of APC, infection with MOPV in vitro can induce a much stronger T-cell response than infection with LASV, again indicating that pathogenic LASV infection likely requires suppression of innate and adaptive immune responses. ${ }^{27}$ The role of humoral responses during LASV infection is less clear. Antibody responses against LASV are typically weak with low levels of IgM and IgG produced during early infection. Antibodies are thought by many to not contribute to natural recovery, as neutralizing antibodies are not produced at high levels. However, the role of antibodies in protection against LF is still not fully clear and mechanisms such as antibody-dependent cell-mediated cytotoxicity, complement fixation/activation, and opsonization may potentially play a role in protection from disease.

Several lines of evidence point to a strong T-cell response to LASV as critical for protection against LF. Defective $\mathrm{T}$-cell responses predominate during severe LASV infection, while strong T-cell responses are typically seen in individuals who can control the infection. In NHP models, undetectable $\mathrm{T}$-cell responses correlate with lethality. In these models, we see decreased proliferation, activation, cytokine production, as well as a lack of T-cell expression of CD25 and CD69, promoting disease progression. ${ }^{13,28}$ As already discussed, the lack of T-cell activation is likely due to an inhibition of the interaction between infected and antigen-primed DCs presenting LASV peptides to T cells. Indeed, LASV-infected DCs are unable to activate CD4 and CD8 T cells in vitro, while DCs infected with MOPV readily activate these cells. ${ }^{27}$ The exact mechanisms of T-cell depletion are not clear; however, the ability of LASV to prevent DC activation likely plays a role. There is also evidence that LASV-specific $\mathrm{T}$ cells can contribute to disease through macrophage activation, resulting in deleterious cell-mediated inflammatory reactions and damage to secondary lymphoid organs. ${ }^{29}$ The role this might play in active suppression of $\mathrm{T}$-cell responses during LASV infection is unclear. This suggests that, while likely important for protection against disease, strong T-cell responses may contribute to pathogenesis and this should be examined further in more appropriate models.

CD4 and CD8 T cells are activated early during LASV infection, and LASV-specific T cells are detected following convalescence despite a lack of LASV-specific antibodies. ${ }^{13,30}$ Strong T-cell responses against both NP and GPC of LASV seem to persist for years following infection. ${ }^{25,26}$ Indirect evidence from vaccination studies also suggests that T-cell immunity is important for protection against LF. Strong T-cell responses against GP1 and GP2 induced by a vacciniavectored LASV vaccine were protective against LASV challenge. ${ }^{31}$ In addition, strong activation and proliferation of CD4 and CD8 T cells following LASV challenge are seen in animals that survive infection. In patients who have recovered from LF, LASV-specific antibody levels are present, but soon become undetectable while LASV-specific memory CD4 T cells persist. There is also evidence that seronegative survivors of LASV do not suffer from LF upon reinfection, likely due to a cell-mediated memory response. ${ }^{26,30,32,33}$ Overall, while the specific phenotypes of responding $\mathrm{T}$ cells and their roles in LASV infection remain to be seen, there is strong evidence suggesting that efficient activation and proliferation of LASV-specific T cells are important for protection against LF.

The role of humoral immune responses during LASV infection has been debated more than that of $\mathrm{T}$ cells. Their correlation with survival has been difficult to determine due to the variability in antibody responses. IgM and $\mathrm{IgG}$ responses occur during LASV infection, but to a lesser degree than what might be expected for them to play a major role in protection from disease. Additionally, neutralizing antibodies against 
LASV are not produced in large quantities during infection due to the structural properties of the LASV GPC and its glycan shield, potentially limiting the role of the humoral response in protection. ${ }^{21}$ Despite these issues, the importance of antibodies in protection against LASV may be understated. The vesicular stomatitis virus vaccine expressing LASV glycoprotein (VSV-LASV-GPC) is able to induce a strong humoral response against LASV GPC and provides similar protection to vaccines inducing strong T-cell responses. ${ }^{28}$ The use of immune plasma and convalescent serum as a treatment for LASV infection in experimental models has shown mixed results, likely contributing to the skepticism of the protective ability of antibodies. ${ }^{34-36}$ However, the use of human monoclonal antibodies in both guinea pig and NHP models of LF has shown to be protective. ${ }^{37,38}$ These monoclonal antibodies used for protection in both models have a high neutralizing index, providing evidence that perhaps a strong neutralizing response may be protective. The lack of neutralizing antibodies produced during natural infection may be the reason why no correlation between antibody production and survival has been observed. However, these animal models show that vaccine/therapeutic candidates are able to induce high neutralizing titers and may be feasible for protection against LASV.

\section{Preclinical vaccine candidates for Lassa fever}

There has been considerable progress made in the development of preclinical vaccine candidates for the prevention of LF since its original description. Some progress has been made with several non-replication-competent vaccines including alphavirus replicons, ${ }^{39,40}$ whole-inactivated LASV, ${ }^{41}$ and DNA electroporation. ${ }^{42-44}$ Additionally, therapeutics have been developed that include the preclinical testing of ribavirin and favipiravir. Currently, the best therapeutic strategy against LASV remains favipiravir, which can be administered up to 9 days post-infection in a guinea pig model (Figure 2B). ${ }^{45}$ Other treatments such as human monoclonal antibodies have been tested in both guinea pigs ${ }^{37}$ and NHPs, ${ }^{38}$ but are only effective when administered very early in the disease course. With this in mind, some of the best candidates with strong efficacy and immunogenicity for LASV prevention have been based on replication-competent vaccine modalities (Table 1 ).

\section{Recombinant vaccinia virus vaccine platform}

In 1987, the first successful vaccine directed against LASV was described. A recombinant vaccinia virus (Lister strain) was engineered to express the NP from LASV and given to outbred Hartley guinea pigs. ${ }^{46}$ Animals that received the recombinant virus showed no signs of disease, and no virus was isolated from the blood after challenge with $1 \times 10^{4}{ }_{\text {TCID50 }}$ of LASV strain GA391 (Nigerian isolate). All control animals succumbed to infection between Days 14 and 16. Interestingly, a similar dose of the prototypical strain Josiah, a Sierra Leone isolate, is generally only $10 \%-20 \%$ lethal in Hartley guinea pigs, which highlights the significant diversity and pathogenic heterogeneity of LF. A second study using a guinea pig-adapted version of Josiah confirmed the protective efficacy of various recombinant strains of vaccinia expressing either LASV NP or GPC with $94 \%$ and $79 \%$ protection, respectively. ${ }^{47}$ In contrast to the initial vaccinia experiments, most animals including the recombinant vaccine vaccinated animals showed some signs of disease with mild fever and acute viremia that was 10-fold less than control animals. Interestingly, a combination of NP and GPC vaccinia viruses led to a poorer outcome with only $58 \%$ protection. A set of secondary comprehensive studies was undertaken in rhesus and cynomolgus macaques to test the individual vaccinia vaccines expressing GP1, GP2, and NP. ${ }^{31}$ Ninety percent of animals that received all three proteins survived a lethal LASV challenge $(n=10)$. Of considerable interest was that all animals that received the individual proteins succumbed to infection (GP1, GP2, or NP; $0 \%$ survival).

Table I Animal testing of replication-competent LASV vaccine candidates

\begin{tabular}{llll}
\hline Vaccine platform & Antigens & Guinea pig efficacy & NHP efficacy \\
\hline Vaccinia virus & NP & $100 \% \%^{46} 94 \%{ }^{47}$ & $0 \%$ cynos; $^{31} 43 \%$ rhesus $^{31}$ \\
& GPI or GP2 & & $0 \%$ cynos $^{31}$ \\
ML29 & GPC & $79 \% ;^{46} 100 \%{ }^{63}$ & $67 \% \mathrm{cynos}^{31} 100 \%$ rhesus $^{31,64}$ \\
YFVI7D & GPC and NP & $100 \%^{51,65}$ & $100 \%$ marmosets \\
& GPC & $80 \%^{53}$ & $0 \%$ marmosets \\
VSV & GPI and GP2 & $83 \%^{54}$ & \\
& NP & $67 \%^{61}$ & $100 \%$ cynos $^{28,61,62}$ \\
\hline
\end{tabular}

Abbreviations: LASV, Lassa virus; NHPs, non-human primates; NP, nucleoprotein; GPC, glycoprotein precursor; YFVI7D, yellow fever I7D; VSV, vesicular stomatitis virus. 
This is in stark contrast to the previous guinea pig experiments and despite all macaques generating robust anti-NP antibody responses. In addition, all animals that received a combination of GP1 and GP2 survived indicating that both proteins are independently integral for protection against lethal disease. These initial studies, combined with the failure of whole-inactivated LASV to protect against lethal infection, ${ }^{41}$ indicate a GPC-specific cell-mediated component to LASV protection.

\section{Reassortant vaccine platform, ML29}

Taking advantage of closely related arenaviruses, another more recent vaccine candidate was designed using gene reassortments from their bisegmented RNA genomes. ${ }^{48}$ A reassortant virus (ML29) was subsequently generated by rationally combining the non-pathogenic L RNA from MOPV and the S RNA from LASV Josiah. ${ }^{49}$ In addition, there were several lab-adapted mutations that led to further attenuation of the ML29 strain. ${ }^{50}$ Studies in strain 13 guinea pigs vaccinated with the ML29 vaccine were completely protected from lethal LASV infection (Josiah and LASV803213 ) up to 30 -days post-vaccination. ${ }^{51}$ Of note, these animals had undetectable virus in the blood and tissues as found by conventional plaque assay and qRT-PCR, indicating that sterilizing immunity was achieved. These initial studies showed that despite the significant genomic heterogeneity between outbreak strains, a universal vaccine for LASV might be achievable. In addition, the ML29 vaccine was able to provide up to $80 \%$ protection when given 2 days after a lethal challenge. ${ }^{51}$ The ML29 vaccine has also undergone some testing in small NHPs, Marmosets in particular, and achieved $100 \%$ protection with accompanying sterilizing immunity. ${ }^{52}$ However, further testing in the gold standard models for LASV disease, rhesus or cynomolgus macaques, is warranted. ML29 also has substantial safety concerns with regards to the genetic stability of a recombinant virus as well as safety in HIV-infected individuals within LASV endemic regions. ${ }^{32}$

\section{Recombinant yellow fever vaccine platform, YFI7D}

Another recombinant vaccine developed by the same research group ${ }^{53}$ makes use of the yellow fever $17 \mathrm{D}$ (YF17D) vaccine, which is one of the safest and most effective vaccines ever developed. The YF17D vaccine is highly immunogenic, induces strong cell-mediated immunity, and tolerates recombinant addition of transgenes into its genome. An initial vaccine developed to express the LASV GPC was able to protect $80 \%$ of strain 13 guinea pigs from lethal LASV challenge. However, the genetic stability of the virus was less than ideal and GPC expression waned over 5 passages. ${ }^{53}$ In an attempt to remedy the genetic instability, separate recombinant viruses were generated expressing GP1 or GP2, as well as attempts to shorten the transgene. ${ }^{54} \mathrm{~A}$ mixture of the two recombinant viruses protected strain 13 guinea pigs from fatal LASV challenge; however, sterilizing immunity was not achieved. Unfortunately, further testing of the YF17D-GPC LASV vaccine in marmosets resulted in poor immunogenicity and no protection from lethal LASV challenge. ${ }^{55}$ While YF17D is a safe vaccine, more development is currently needed to achieve an efficacious vaccine for both yellow fever and LASV.

\section{Recombinant vesicular stomatitis virus vaccine platform, VSV}

Despite the considerable list of preclinical candidates for LASV prevention, none have yet shown combined efficacy and safety in humans. In 2013, an unprecedented Ebola outbreak in West Africa resulted in over 30,000 documented cases of infection and $>10,000$ deaths. As an emergency measure, several vaccines were accelerated into Phase III clinical trials for testing during the outbreak. One of the most successful vaccines to show $100 \%$ efficacy in a ring vaccination trial was recombinant vesicular stomatitis virus expressing Ebola virus GPC (VSV-EBOV-GPC). ${ }^{56}$ Antibodies have been implicated as the main correlate of protection for EBOV infection ${ }^{57}$ with respect to VSVEBOV-GPC vaccination, and has been shown to be long lasting for up to 14 months in the NHP model. ${ }^{58}$ However, multiple lines of evidence suggest that the main correlate of protection for LASV involves cell-mediated immunity despite recent studies indicating that monoclonal antibody therapy is sufficient for protection..$^{37,38}$ The recombinant VSV system is able to tolerate the replacement of the VSV glycoprotein with foreign virus glycoproteins. The resulting recombinant viruses are attenuated, safe, and highly immunogenic, making this vaccine a strong candidate for LASV prevention. In 2004, the first description of VSV expressing LASV GPC was published and was shown to be apathogenic in mice. ${ }^{59} \mathrm{~A}$ follow-up study by the same group characterized the VSV-LASV-GPC vaccine in NHPs. ${ }^{28}$ Cynomolgus macaques were vaccinated with a single dose of VSV-LASV-GPC $\left(2 \times 10^{7} \mathrm{PFU}\right)$ and challenged 28 days later with a lethal dose of Josiah. All vaccinated animals survived the challenge with no overt signs of disease or changes in blood chemistry or hematology. 
There is considerable genetic variability between strains of LASV, which cluster into at least five clades. Clades I-III are generally localized to Nigeria, while clade IV and V viruses were isolated from Sierra Leonne, Guinea, Liberia, and Mali. Renewed studies almost 10 years after the initial characterization of the VSV-LASV-GPC vaccine sought to test whether it could act as a universal vaccine against genetically distinct LASV isolates. Geographically distinct isolates have been shown to have drastically altered disease manifestations and thus may not be covered by the VSVLASV-GPC vaccine originally constructed with Josiah GPC ${ }^{60}$ Safronetz et al discovered that the single Josiah-based VSV vaccine could provide $100 \%$ protection against several isolates including Sierra Leone (Josiah; clade IV), Liberia (Z-132; clade IV), Mali (Soromba-R; clade V), and Nigeria (Pinneo; clade I) in strain 13 guinea pigs, identifying VSVLASV-GPC as a universal vaccine candidate. Additionally, cynomolgus macaques vaccinated with VSV-LASV-GPC showed no overt signs of disease when challenged with the Liberian isolate ${ }^{61}$ The current Nigerian outbreak strain of LASV is thought to be a clade III isolate and has yet to be tested for efficacy against the VSV-LASV-GPC vaccine. These data lend strong support to the possibility of a universal LASV vaccine capable of being effective against all lineages in the region.

With the potential of widespread use of VSV-EBOV in the wake of the West African Ebola outbreak, an important aspect to consider would be the potential for an increase in pre-existing immunity to the VSV backbone. A deployment of a second VSV-based vaccine in the region, mainly VSVLASV-GPC, may not provide adequate immunogenicity and protection. Marzi et al sought to address this concern by designing consecutive LASV and EBOV studies in cynomolgus macaques. ${ }^{62}$ Initially, three macaques were vaccinated with VSV-LASV-GPC and then challenged with a lethal dose of LASV Josiah. All three animals survived the challenge with no overt signs of disease and no virus was detected in the blood. Additionally, two animals showed no signs of seroconversion after challenge, suggesting near-sterilizing immunity. A single control animal was infected in parallel, which rapidly succumbed to classical LASV disease on Day 13. The same three surviving animals were then vaccinated with a dose of VSV-EBOV-GPC and subsequently challenged with a lethal dose of EBOV 28 days later. Despite having significant VSV antibody titers prior to VSV-EBOVGPC vaccination, all immunized animals survived the challenge, while a single control animal succumbed to EBOV disease on Day 7. More importantly, this study shows that the VSV vector has the potential to be used repeatedly for the protection of both EBOV and LASV infection in Africa. The VSV recombinant system is extremely robust and has the potential to express and tolerate the expression of multiple transgenes. The combination of both EBOV and LASV glycoprotein expression in a single vaccine is feasible and has significant potential to prevent future outbreaks of EBOV and LASV in West Africa.

\section{Conclusion}

Unfortunately, despite the best efforts of CEPI taking the lead and preparing for future outbreaks, the current resurgence of LASV in Nigeria has again caught the international community as ill prepared. The recombinant VSV-LASV-GPC vaccine is among one of the leading candidates developed thus far and should be targeted for accelerated development by CEPI and its collaborating partners. There are several important questions that need to be rapidly addressed in the face of this new outbreak. Mainly, has the genetic makeup of the virus undergone a significant change allowing it to spread more readily or become more pathogenic? It will also be important to characterize the new Nigerian outbreak strain of LASV and test the efficacy of these preclinical vaccine candidates, in particular the VSV-LASV-GPC vaccine. As LASV virus is thrust into the international spotlight, it continues to be a neglected emerging pathogen of critical importance. However, with strong clinical candidates poised to be tested in Phase III trials, a preventative vaccine could soon be approved for use in the region.

\section{Disclosure}

The authors report no conflicts of interest in this work.

\section{References}

1. Roberts L. Nigeria hit by unprecedented Lassa fever outbreak. Science. 2018;359(6381):1201-1202.

2. Frame JD, Baldwin JM Jr, Gocke DJ, Troup JM. Lassa fever, a new virus disease of man from West Africa. I. Clinical description and pathological findings. Am J Trop Med Hyg. 1970;19(4):670-676.

3. Monath TP, Newhouse VF, Kemp GE, Setzer HW, Cacciapuoti A. Lassa virus isolation from Mastomys natalensis rodents during an epidemic in Sierra Leone. Science. 1974;185(4147):263-265.

4. Schlie K, Maisa A, Lennartz F, Ströher U, Garten W, Strecker T. Characterization of Lassa virus glycoprotein oligomerization and influence of cholesterol on virus replication. J Virol. 2010;84(2):983-992.

5. McCormick JB, King IJ, Webb PA, et al. A case-control study of the clinical diagnosis and course of Lassa fever. J Infect Dis. 1987;155(3): 445-455

6. Bausch DG, Hadi CM, Khan SH, Lertora JJ. Review of the literature and proposed guidelines for the use of oral ribavirin as postexposure prophylaxis for Lassa fever. Clin Infect Dis. 2010;51(12):1435-1441.

7. Macher AM, Wolfe MS. Historical Lassa fever reports and 30-year clinical update. Emerg Infect Dis. 2006;12(5):835-837. 
8. McCormick JB. Epidemiology and control of Lassa fever. Curr Top Microbiol Immunol. 1987;134:69-78.

9. Baize S, Kaplon J, Faure C, Pannetier D, Georges-Courbot MC, Deubel V. Lassa virus infection of human dendritic cells and macrophages is productive but fails to activate cells. J Immunol. 2004; 172(5):2861-2869.

10. Baize S, Pannetier D, Faure C, et al. Role of interferons in the control of Lassa virus replication in human dendritic cells and macrophages. Microbes Infect. 2006;8(5):1194-1202.

11. Pannetier D, Faure C, Georges-Courbot MC, Deubel V, Baize S. Human macrophages, but not dendritic cells, are activated and produce alpha/beta interferons in response to Mopeia virus infection. $J$ Virol. 2004;78(19):10516-10524.

12. Jahrling PB, Hesse RA, Eddy GA, Johnson KM, Callis RT, Stephen EL. Lassa virus infection of rhesus monkeys: pathogenesis and treatment with ribavirin. J Infect Dis. 1980;141(5):580-589.

13. Baize $S$, Marianneau P, Loth $P$, et al. Early and strong immune responses are associated with control of viral replication and recovery in lassa virusinfected cynomolgus monkeys. J Virol. 2009;83(11):5890-5903.

14. Yun NE, Poussard AL, Seregin AV, et al. Functional interferon system is required for clearance of lassa virus. J Virol. 2012;86(6):3389-3392.

15. Oestereich L, Lüdtke A, Ruibal P, et al. Chimeric mice with competent hematopoietic immunity reproduce key features of severe lassa fever. PLoS Pathog. 2016;12(5):e1005656.

16. Yun NE, Seregin AV, Walker DH, et al. Mice lacking functional STAT1 are highly susceptible to lethal infection with Lassa virus. $J$ Virol. 2013;87(19):10908-10911.

17. Xing J, Ly H, Liang Y. The Z proteins of pathogenic but not nonpathogenic arenaviruses inhibit RIG-I-like receptor-dependent interferon production. J Virol. 2015;89(5):2944-2955.

18. Pythoud C, Rodrigo WW, Pasqual G, et al. Arenavirus nucleoprotein targets interferon regulatory factor-activating kinase IKKe. $J$ Virol. 2012;86(15):7728-7738.

19. Rodrigo WW, Ortiz-Riaño E, Pythoud C, Kunz S, de la Torre JC, Martínez-Sobrido L. Arenavirus nucleoproteins prevent activation of nuclear factor kappa B. J Virol. 2012;86(15):8185-8197.

20. Hastie KM, King LB, Zandonatti MA, Saphire EO. Structural basis for the dsRNA specificity of the Lassa virus NP exonuclease. PLoS One. 2012;7(8):e44211.

21. Jiang X, Huang Q, Wang W, et al. Structures of arenaviral nucleoproteins with triphosphate dsRNA reveal a unique mechanism of immune suppression. J Biol Chem. 2013;288(23):16949-16959.

22. Prescott JB, Marzi A, Safronetz D, Robertson SJ, Feldmann H, Best SM. Immunobiology of Ebola and Lassa virus infections. Nat Rev Immunol. 2017;17(3):195-207.

23. Russier M, Pannetier D, Baize S. Immune responses and Lassa virus infection. Viruses. 2012;4(11):2766-2785.

24. Carrion R Jr, Brasky K, Mansfield K, et al. Lassa virus infection in experimentally infected marmosets: liver pathology and immunophenotypic alterations in target tissues. $J$ Virol. 2007;81(12):6482-6490.

25. Meulen Jt, Badusche M, Satoguina J, et al. Old and New World arenaviruses share a highly conserved epitope in the fusion domain of the glycoprotein 2, which is recognized by Lassa virus-specific human CD4+ T-cell clones. Virology. 2004;321(1):134-143.

26. ter Meulen J, Badusche M, Kuhnt K, et al. Characterization of human CD4(+) T-cell clones recognizing conserved and variable epitopes of the Lassa virus nucleoprotein. J Virol. 2000;74(5):2186-2192.

27. Pannetier D, Reynard S, Russier M, et al. Human dendritic cells infected with the nonpathogenic Mopeia virus induce stronger T-cell responses than those infected with Lassa virus. J Virol. 2011;85(16): 8293-8306.

28. Geisbert TW, Jones S, Fritz EA, et al. Development of a new vaccine for the prevention of Lassa fever. PLoS Med. 2005;2(6):e183.

29. Flatz L, Rieger T, Merkler D, et al. T cell-dependence of Lassa fever pathogenesis. PLoS Pathog. 2010;6(3):e1000836.
30. Günther $\mathrm{S}$, Kühle $\mathrm{O}$, Rehder D, et al. Antibodies to Lassa virus $\mathrm{Z}$ protein and nucleoprotein co-occur in human sera from Lassa fever endemic regions. Med Microbiol Immunol. 2001;189(4):225-229.

31. Fisher-Hoch SP, Hutwagner L, Brown B, McCormick JB. Effective vaccine for lassa fever. $J$ Virol. 2000;74(15):6777-6783.

32. Lukashevich IS, Pushko P. Vaccine platforms to control Lassa fever. Expert Rev Vaccines. 2016;15(9):1135-1150.

33. Fisher-Hoch SP, McCormick JB. Lassa fever vaccine. Expert Rev Vaccines. 2004;3(2):189-197.

34. Frame JD, Verbrugge GP, Gill RG, Pinneo L. The use of Lassa fever convalescent plasma in Nigeria. Trans $R$ Soc Trop Med Hyg. 1984;78(3):319-324.

35. Jahrling PB, Peters CJ. Passive antibody therapy of Lassa fever in cynomolgus monkeys: importance of neutralizing antibody and Lassa virus strain. Infect Immun. 1984;44(2):528-533.

36. Jahrling PB, Peters CJ, Stephen EL. Enhanced treatment of Lassa fever by immune plasma combined with ribavirin in cynomolgus monkeys. J Infect Dis. 1984;149(3):420-427.

37. Cross RW, Mire CE, Branco LM, et al. Treatment of Lassa virus infection in outbred guinea pigs with first-in-class human monoclonal antibodies. Antiviral Res. 2016;133:218-222.

38. Mire CE, Cross RW, Geisbert JB, et al. Human-monoclonal-antibody therapy protects nonhuman primates against advanced Lassa fever. Nat Med. 2017;23(10):1146-1149.

39. Pushko P, Geisbert J, Parker M, Jahrling P, Smith J. Individual and bivalent vaccines based on alphavirus replicons protect guinea pigs against infection with Lassa and Ebola viruses. $J$ Virol. 2001; 75(23):11677-11685.

40. Wang M, Jokinen J, Tretyakova I, Pushko P, Lukashevich IS. Alphavirus vector-based replicon particles expressing multivalent cross-protective Lassa virus glycoproteins. Vaccine. 2018;36(5):683-690.

41. McCormick JB, Mitchell SW, Kiley MP, Ruo S, Fisher-Hoch SP. Inactivated Lassa virus elicits a non protective immune response in rhesus monkeys. J Med Virol. 1992;37(1):1-7.

42. Cashman KA, Broderick KE, Wilkinson ER, et al. Enhanced efficacy of a codon-optimized DNA vaccine encoding the glycoprotein precursor gene of Lassa virus in a guinea pig disease model when delivered by dermal electroporation. Vaccines (Basel). 2013;1(3):262-277.

43. Cashman KA, Wilkinson ER, Shaia CI, et al. A DNA vaccine delivered by dermal electroporation fully protects cynomolgus macaques against Lassa fever. Hum Vaccin Immunother. 2017;13(12): 2902-2911.

44. Cashman KA, Wilkinson ER, Wollen SE, et al. DNA vaccines elicit durable protective immunity against individual or simultaneous infections with Lassa and Ebola viruses in guinea pigs. Hum Vaccin Immunother. 2017;13(12):3010-3019.

45. Safronetz D, Rosenke K, Westover JB, et al. The broad-spectrum antiviral favipiravir protects guinea pigs from lethal Lassa virus infection post-disease onset. Sci Rep. 2015;5:14775.

46. Clegg JC, Lloyd G. Vaccinia recombinant expressing Lassa-virus internal nucleocapsid protein protects guinea pigs against Lassa fever. Lancet. 1987;2(8552):186-188.

47. Morrison HG, Bauer SP, Lange JV, Esposito JJ, McCormick JB, Auperin DD. Protection of guinea pigs from Lassa fever by vaccinia virus recombinants expressing the nucleoprotein or the envelope glycoproteins of Lassa virus. Virology. 1989;171(1):179-188.

48. Lukashevich IS, Vasiuchkov AD, Stel'makh TA, Scheslenok EP, Shabanov AG. [The isolation and characteristics of reassortants between the Lassa and Mopeia arenaviruses]. Vopr Virusol. 1991;36(2):146-150. Russian [with English abstract].

49. Lukashevich IS. Generation of reassortants between African arenaviruses. Virology. 1992;188(2):600-605.

50. Moshkoff DA, Salvato MS, Lukashevich IS. Molecular characterization of a reassortant virus derived from Lassa and Mopeia viruses. Virus Genes. 2007;34(2):169-176. 
51. Carrion R Jr, Patterson JL, Johnson C, et al. A ML29 reassortant virus protects guinea pigs against a distantly related Nigerian strain of Lassa virus and can provide sterilizing immunity. Vaccine. 2007;25(20):4093-4102.

52. Lukashevich IS, Carrion R Jr, Salvato MS, et al. Safety, immunogenicity, and efficacy of the ML29 reassortant vaccine for Lassa fever in small non-human primates. Vaccine. 2008;26(41):5246-5254.

53. Bredenbeek PJ, Molenkamp R, Spaan WJ, et al. A recombinant Yellow Fever 17D vaccine expressing Lassa virus glycoproteins. Virology. 2006;345(2):299-304.

54. Jiang X, Dalebout TJ, Bredenbeek PJ, et al. Yellow fever 17Dvectored vaccines expressing Lassa virus GP1 and GP2 glycoproteins provide protection against fatal disease in guinea pigs. Vaccine. 2011;29(6):1248-1257.

55. Lukashevich IS. Advanced vaccine candidates for Lassa fever. Viruses 2012;4(11):2514-2557.

56. Henao-Restrepo AM, Longini IM, Egger M, et al. Efficacy and effectiveness of an rVSV-vectored vaccine expressing Ebola surface glycoprotein: interim results from the Guinea ring vaccination clusterrandomised trial. Lancet. 2015;386(9996):857-866.

57. Marzi A, Engelmann F, Feldmann F, et al. Antibodies are necessary for $\mathrm{rVSV} / \mathrm{ZEBOV-GP-mediated} \mathrm{protection} \mathrm{against} \mathrm{lethal} \mathrm{Ebola} \mathrm{virus}$ challenge in nonhuman primates. Proc Natl Acad Sci U S A. 2013; 110(5):1893-1898.

58. Mire CE, Geisbert JB, Agans KN, et al. Durability of a vesicular stomatitis virus-based marburg virus vaccine in nonhuman primates. PLoS One. 2014;9(4):e94355.

59. Garbutt M, Liebscher R, Wahl-Jensen V, et al. Properties of replicationcompetent vesicular stomatitis virus vectors expressing glycoproteins of filoviruses and arenaviruses. J Virol. 2004;78(10):5458-5465.
60. Safronetz D, Strong JE, Feldmann F, et al. A recently isolated Lassa virus from Mali demonstrates atypical clinical disease manifestations and decreased virulence in cynomolgus macaques. J Infect Dis. 2013; 207(8):1316-1327.

61. Safronetz D, Mire C, Rosenke K, et al. A recombinant vesicular stomatitis virus-based Lassa fever vaccine protects guinea pigs and macaques against challenge with geographically and genetically distinct Lassa viruses. PLoS Negl Trop Dis. 2015;9(4):e0003736.

62. Marzi A, Feldmann F, Geisbert TW, Feldmann H, Safronetz D. Vesicular stomatitis virus-based vaccines against Lassa and Ebola viruses. Emerg Infect Dis. 2015;21(2):305-307.

63. Auperin DD, Esposito JJ, Lange JV, et al. Construction of a recombinant vaccinia virus expressing the Lassa virus glycoprotein gene and protection of guinea pigs from a lethal Lassa virus infection. Virus Res. 1988;9(2-3):233-248.

64. Fisher-Hoch SP, McCormick JB, Auperin D, et al. Protection of rhesus monkeys from fatal Lassa fever by vaccination with a recombinant vaccinia virus containing the Lassa virus glycoprotein gene. Proc Natl Acad Sci US A. 1989;86(1):317-321.

65. Lukashevich IS, Patterson J, Carrion R, et al. A live attenuated vaccine for Lassa fever made by reassortment of Lassa and Mopeia viruses. J Virol. 2005;79(22):13934-13942.

66. World Health Organization. 2018. World Health Organization Lassa Fever Nigeria Report. Available from: http://www.who.int/csr/don/23march-2018-lassa-fever-nigeria/en/. Accessed April 07, 2018.
Drug Design, Development and Therapy

\section{Publish your work in this journal}

Drug Design, Development and Therapy is an international, peerreviewed open-access journal that spans the spectrum of drug design and development through to clinical applications. Clinical outcomes, patient safety, and programs for the development and effective, safe, and sustained use of medicines are the features of the journal, which

\section{Dovepress}

has also been accepted for indexing on PubMed Central. The manuscript management system is completely online and includes a very quick and fair peer-review system, which is all easy to use. Visit http://www.dovepress.com/testimonials.php to read real quotes from published authors.

Submit your manuscript here: http://www.dovepress.com/drug-design-development-and-therapy-journal 